\title{
Do depressive symptoms correlate with oxidative stress in a sample of healthy college students?
}

\author{
Masateru Matsushita ${ }^{*, * *, * \star *}$ \\ Takayuki Kumano-Go ${ }^{*, * *, * * *}$ \\ Nakamori Suganuma*,**,*** \\ Hiroyoshi Adachi ${ }^{*, * *, * * *}$ \\ Schubei Yamanura*,*** \\ Hiroko Morishima ${ }^{*, * \star *}$ \\ Yoshihisa Shigedo*,*** \\ Akira Mikami i, ${ }^{* \star *, * \star \star}$ \\ Masatoshi Takeda* \\ Yoshiro Sugita ${ }^{*, * *, * * *}$ \\ * Department of Psychiatry, Osaka University \\ Graduate School of Medicine, Suita \\ ** Osaka University Health Care Center, \\ Osaka, Toyonaka \\ *** Sleep Medical Center, Osaka University \\ Hospital, Suita \\ JAPAN
}

\begin{abstract}
Background and Objectives: Major depression and sub-threshold depressive symptoms are associated with health crisis. Oxidative stress may be a mechanism for major depression. In the present study, we examined the relationship between the degree of depressive symptoms and oxidative status using a reliable and inexpensive method that evaluates endogenous hydroperoxides.

Methods: We conducted a cross-sectional study in 54 non-smoking college students and measured serum reactive oxygen metabolites (ROMs) and the biological antioxidant potential (BAP) as an index of oxidative status. Depressive symptoms were assessed by the Beck Depression Inventory (BDI).

Results: The concentrations of ROMs did not differ between the lower BDI group (BDI $<14)$ and the higher BDI group (BDI $\geq 14)(282.7 \pm 59.84$ U.CARR vs $307.7 \pm 67.51$ U.CARR, $z=-1.19, P=0.239)$. We did find a significant relationship between ROM concentration values and higher BDI scores $(r h o=0.30, P=0.042)$. BAP levels in the higher BDI group were not significantly greater than those in the lower BDI group $(z=-0.108$, $P=0.287$ ). There was no significant correlation between BAP and depressive symptoms ( $r h o=0.22, P=0.140$ ). Moreover, we conducted a multiple regression analysis to control for gender difference and difference in sleep perception of the previous night between the two BDI groups. However, depressive symptoms were not significantly predicted by ROM concentrations $(\beta=0.28, P=0.076)$.
\end{abstract}


Conclusions: While results of the present study demonstrated a slight correlation between depressive symptoms and oxidative stress, this linkage could not be confirmed after controlling for significant confounding factors. This result should be verified in a larger sample.

Received: 22 December 2008

Revised: 20 December 2009

Accepted: 4 February 2010

\section{Introduction}

Major depression is serious public health problems that increase the risk of comorbid diseases such as cardiovascular disease ${ }^{1-3}$, stroke $^{4}$, cancer ${ }^{5-7}$ and diabetes ${ }^{8-10}$. Oxidative stress has gained attention in psychiatric medicine, and various markers of oxidative stress have been identified ${ }^{11}$. Recently, reactive oxygen metabolites (ROMs) and the biological antioxidant potential (BAP) were used to evaluate oxidative status, and their significance as clinical markers has been reported in various medical fields ${ }^{12-15}$. Although the increase in lipid peroxidation products and elevated oxidative DNA damage have been demonstrated in depressed subjects $^{16-18}$, the ROMs and BAP have not been examined. We, therefore, aimed to examine the relationship between depressive symptoms and oxidative stress, using the ROMs and BAP.

\section{Methods}

\section{Participants and procedure}

Participants were 54 non-smoking students. These volunteers were excluded from analysis if they reported a history of psychiatric disorders, an irregular sleep-wake rhythm, taking a supplement, or drinking al- coholic beverages almost daily. None of those included in the analysis had sought medical care in the month previous to the study. Female participants were excluded from analysis if they were menstruating. Forty-seven participants were included in the data analysis.

We asked participants to maintain regular hours for bedtime and awakening, and to abstain from eating and drinking except for water and tea after the usual dinner hour. On the following day between 9:00 am and 12:00 noon we obtained a fasting blood sample and assessed depressive symptoms.

Subjects were asked to complete self-administered questionnaires, and answered items on the lifestyle as well as other characteristics such as age, gender, menstrual state, height and weight, and history of psychiatric disorders. Depressive symptoms were assessed by the Beck Depression Inventory-II (BDI), which consists of 21 items. Scores were scaled from 0 to 63 , with higher scores indicating greater depressive symptoms. Total scores can be classified as indicating four severity categories: minimal (0-13), mild (14-19), moderate (20-28), and severe $(29-63)^{19}$. Participants were classified into two groups according to BDI scores: a lower BDI group (0-13) and a higher BDI group (14-39). Questions were also asked regarding personality and anxiety. These findings are to be reported in a separate paper. 
Table I

Characteristics of the study subjects

\begin{tabular}{lccc} 
& $\begin{array}{c}\text { Lower BDI group } \\
n=31\end{array}$ & $\begin{array}{c}\text { Higher BDI group } \\
n=16\end{array}$ & $P$ value \\
\hline Age years & $20.7 \pm 0.70$ & $20.8 \pm 0.91$ & 0.859 \\
\hline Male, $n(\%)$ & $17(54.8)$ & $4(25.0)$ & 0.068 \\
\hline Body mass index & $21.5 \pm 2.96$ & $20.8 \pm 2.87$ & 0.262 \\
\hline Sleep time, previous night (hour) & $6.7 \pm 0.77$ & $6.2 \pm 1.28$ & 0.265 \\
\hline $\begin{array}{l}\text { Perception of sleep, previous night, } n(\%) \\
\text { Soundly }\end{array}$ & $26(83.9)$ & $8(50)$ & $0.020^{*}$ \\
Poor & $5(16.1)$ & $8(50)$ & \\
\hline Alcohol consumption, $n(\%)^{+}$ & & & \\
$\quad$ Sometimes & $6(19.4)$ & $5(31.3)$ & 0.472 \\
$\quad$ Never & $25(80.6)$ & $11(68.8)$ & \\
\hline Engages in exercice, $n(\%)^{+}$ & & \\
$\quad$ Sometimes & $9(29.0)$ & $6(37.5)$ & 0.742 \\
None & $22(71.0)$ & $10(62.5)$ & \\
\hline
\end{tabular}

Note: ${ }^{+}$No one asnswered "almost every day".

$* P<0.05$.

\section{Assay of oxidative status}

ROMs were evaluated by the d-ROMs test (Diacron International, Grosseto, Italy) as an index of oxidative stress, which estimates endogenous hydroperoxide. The $10 \mu \mathrm{l}$ of Blood was collected from all participants by finger puncture with disposable lancets and analyzed with the free radical analysis system (FRAS) immediately after collection. The results of the d-ROM test are expressed in arbitrary units called "Carratelli Units" (U.CARR).

The BAP test was performed by the FRAS system, which includes both a photometric device and an incorporated thermostatic mini-centrifuge. This test measures the blood concentration of antioxidants as agents able to reduce iron from its ferric $\left(\mathrm{Fe}^{3+}\right)$ to ferrous form $\left(\mathrm{Fe}^{2+}\right)$. We estimated the intensity of this chromatic change photometrically. The results are expressed as $\mu \mathrm{mol} / \mathrm{l}$.

\section{Statistical analysis}

In this study, we used the Mann-Whitney $U$ test, chi-square test, and Spearman's correlation analysis. Additionally, if we found a significant linkage, multiple regression analysis was applied to assess the association of the depressive symptoms with potential confounding factors.

\section{Results}

The demographic data and results are summarized in Table I. There was a difference in perception of the previous night's sleep. Regarding the possible effects of gender differences in results of assessment of ROMs and BAP, female students had a higher value for ROMs compared to males $(z=$ $-3.18, P=0.001)$, but there was no difference in the BAP value $(z=-0.77, P=0.438)$. 
Participants in the higher BDI group had a mean ROM level of $307.7 \pm 67.51$ U.CARR and those in the lower BDI group had a mean ROM level of $282.7 \pm 59.84$ U.CARR (Table II), with no significant difference between the two groups. Likewise, the mean BAP level in the higher BDI group was not significantly higher than that in the lower group, and no significant correlation was observed between the BDI score and BAP $(r h o=0.22, P=0.140)$. However, we found a weak correlation between the BDI score and the ROM concentration ( $r h o=0.30$, $P=0.042$ ). Figure 1 shows a scatter plot of the relationship between oxidative stress and depressive symptoms. Only in female participants there was a statistically slight relationship between depressive symptoms and ROMs. Because between-groups differences in gender and the perception of sleep were found, we applied multivariate analysis to control the effect for of these differences statistically. As shown in Table III, although the BDI score appeared to have a relationship with the ROM value after control for the confounding factors, the probability did not reach the $5 \%$ level of statistical significance.

Table II

Comparisons of oxidative indexes in lower and higher BDI groups

\begin{tabular}{lclcccl} 
& Lower BDI group & & Higher BDI group & & $P$ value \\
\hline Oxidative indexes & & & & & & \\
$\quad$ & $282.7 \pm 59.84$ & vs & $307.7 \pm 67.51$ & 0.239 & n.s \\
ROMs & $1890.7 \pm 703.93$ & vs & $2210.1 \pm 857.18$ & 0.287 & n.s \\
BAP & &
\end{tabular}

Note: ROMs, Reactive Oxygen Metabolites; BAP, Biological Antioxidant Potential.

Values express mean and standard deviation.

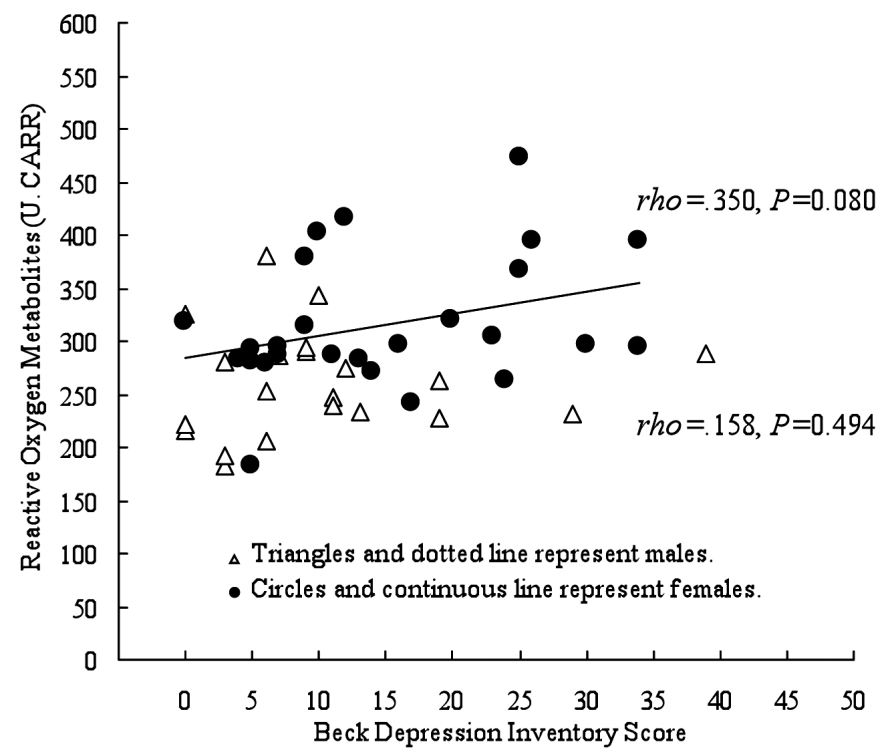

Figure 1. Correlations between reactive oxygen metabolites and the Beck depression inventory score according to gender were shown separately. 


\section{Conclusions}

In this study, we found that depressive symptoms had a slight correlation with the increased concentration of ROMs, although no significant differences in the levels of ROMs and BAP between the two BDI groups were observed. Previous studies have demonstrated a significant correlation between oxidative stress and depression ${ }^{16-18,20,21}$. Furthermore, Bilici et al. reported that lipid peroxidation and antioxidative enzyme levels were significantly decreased to normal levels after selective serotonin reuptake inhibitor therapy for 12 weeks ${ }^{22}$. However, our results showed no clear correlation between depressive symptoms and oxidative status. This may be due to the relatively mild depressive symptoms in our subjects.

There is a possible mechanism that links the oxidative state with depressive symp- toms. Hypothalamic-pituitary-adrenal (HPA) axis overactivity is frequently observed in those with major depression ${ }^{23}$. A HPA axis stimulated by psychological stress may enhance the production of reactive oxygen species relevant to cytotoxicity ${ }^{24}$. Moreover, depression has been related to increased immune activity. Leukocytes, monocytes, T helper cells, and interleukin are immune inflammatory markers that are increased in depressive disorders ${ }^{25-28}$.

The d-ROMs and BAP tests, which are minimally invasive, easy and inexpensive, provide information on the general wellness state of the body for the clinician. To our knowledge, this study is the first attempt to examine an association between depressive symptoms and ROMs. Additional studies that include a larger number of participants and subjects with major depression disorders are needed.

Table III

Multiple linear regression analysis predicting depresive smptoms

\begin{tabular}{lccc} 
& $\beta$ & $t$ & $P$ \\
\hline Gender $($ male $=0$, female $=1)$ & 0.07 & 0.45 & 0.652 \\
\hline Perception of previous night's sleep (soundly $=0$, poor $=1)$ & 0.20 & 1.40 & 0.169 \\
\hline Reactive Oxygen Metabolites & 0.28 & 1.82 & 0.076 \\
\hline
\end{tabular}

Note: ROMs, Reactive Oxygen Metabolites; BAP, Biological Antioxidant Potential.

Values express mean and standard deviation.

\section{Acknowledgments}

This study was supported by a Grant-inaid for Young Scientists (B), (No. 18700552) from the Ministry of Education, Science, Sports and Culture of Japan.

\section{References}

1. Musselman DL, Evans DL, Nemeroff CB. The relationship of depression to cardiovascular disease: epidemiology, biology, and treatment. Arch Gen Psychiatry 1998; 55(7): 580-592.

2. Rudisch B, Nemeroff CB. Epidemiology of comorbid coronary artery disease and depression. Biol Psychiatry 2003; 54(3): 227-240. 
3. Lett HS, Blumenthal JA, Babyak MA, Sherwood A, Strauman T, Robins C, et al. Depression as a risk factor for coronary artery disease: evidence, mechanisms, and treatment. Psychosom Med 2004; 66(3): 305-315.

4. Larson SL, Owens PL, Ford D, Eaton W. Depressive disorder, dysthymia, and risk of stroke: thirteen-year follow-up from the Baltimore epidemiologic catchment area study. Stroke 2001; 32(9): 1979-1983.

5. Penninx BW, Guralnik JM, Pahor M, Ferrucci L, Cerhan JR, Wallace RB, et al. Chronically depressed mood and cancer risk in older persons. J Natl Cancer Inst 1998; 90(24): 1888-1893.

6. Raison CL, Miller AH. Depression and cancer: new developments regarding diagnosis and treatment. Biol Psychiatry 2003; 54(3): 283-294.

7. Spiegel D, Giese-Davis J. Depression and cancer: mechanisms and disease progression. Biol Psychiatry 2003; 54(3): 269-282.

8. Musselman DL, Betan E, Larsen H, Phillips LS. Relationship of depression to diabetes type 1 and 2: epidemiology, biology, and treatment. Biol Psychiatry 2003; 54(3): 317-329.

9. Eaton WW. Epidemiologic evidence on the comorbidity of depression and diabetes. J Psychosom Res 2002; 53(4): 903-906.

10. Eaton WW, Armenian H, Gallo J, Pratt L, Ford DE. Depression and risk for onset of type II diabetes. A prospective population-based study. Diabetes Care 1996; 19(10): 1097-1102.

11. Ng F, Berk M, Dean O, Bush AI. Oxidative stress in psychiatric disorders: evidence base and therapeutic implications. Int J Neuropsychopharmacol 2008; 11(6): 851-876.

12. Atabek ME, Vatansev H, Erkul I. Oxidative stress in childhood obesity. J Pediatr Endocrinol Metab 2004; 17(8): 1063-1068.

13. Digiesi V, Oliviero C, Giannò V, Rossetti M, Fiorillo C, Oradei A, et al. Reactive metabolites of oxygen, lipid peroxidation, total antioxidant capacity and vitamin $\mathrm{E}$ in essential arterial hypertension. Clin Ter 1997; 148(11): 515-519.

14. Katsabeki-Katsafli A, Kerenidi T, Kostikas K, Dalaveris E, Kiropoulos TS, Gogou E, et al. Serum vascular endothelial growth factor is related to systemic oxidative stress in patients with lung cancer. Lung Cancer 2008; 60(2): 271-276.
15. Cesarone MR, Belcaro G, Carratelli M, Cornelli U, De Sanctis MT, Incandela L, et al. A simple test to monitor oxidative stress. Int Angiol 1999; 18(2): 127-130.

16. Yanik M, Erel O, Kati M. The relationship between potency of oxidative stress and severity of depression. Acta Neuropsychiatrica 2004; 16(4): 200-203.

17. Irie M, Miyata M, Kasai H. Depression and possible cancer risk due to oxidative DNA damage. J Psychiatr Res 2005; 39(6): 553-560.

18. Irie M, Asami S, Ikeda M, Kasai H. Depressive state relates to female oxidative DNA damage via neutrophil activation. Biochem Biophys Res Commun 2003; 331(4): 1014-1018.

19. Kojima M, Furukawa TA, Takahashi H, Kawai M, Nagaya T, Tokudome S. Cross-cultural validation of the Beck Depression Inventory-II in Japan. Psychiatry Res 2002; 110(3): 291-299.

20. Forlenza MJ, Miller GE. Increased serum levels of 8-hydroxy-2'-deoxyguanosine in clinical depression. Psychosom Med 2006; 68(1): 1-7.

21. Tsuboi H, Shimoi K, Kinae N, Oguni I, Hori R, Kobayashi F. Depressive symptoms are independently correlated with lipid peroxidation in a female population: comparison with vitamins and carotenoids. J Psychosom Res 2004; 56(1): 53-58.

22. Bilici M, Efe H, Köroğlu MA, Uydu HA, Bekaroğlu M, Değer O. Antioxidative enzyme activities and lipid peroxidation in major depression: alterations by antidepressant treatments. J Affect Disord 2001; 64(1): 43-51.

23. Ansseau M. Diagnostice and therapeutic usefulness of biological markers of depression. University of Liège, Thèse d'Agrégation; 1992.

24. Kasckow JW, Baker D, Geracioti TD Jr. Corticotropin-releasing hormone in depression and post-traumatic stress disorder. Peptides 2001; 22(5): 845-851.

25. Maes M, Lambrechts J, Bosmans E, Jacobs J, Suy E, Vandervorst C, et al. Evidence for a systemic immune activation during depression, results of leukocyte enumeration by flow cytometry in conjunction with monoclonal antibody staining. Psychol Med 1992; 22(1): 45-53.

26. Değer O, Bekaroğlu M, Orem A, Orem S, Uluutku N, Soylu C. Polymorphonuclear (PMN) elastase levels in depressive disorders. Biol Psychiatry 1996; 39(5): 357363. 
27. Seidel A, Arolt V, Hunstiger M, Rink L, Behnisch A, Kirchner H. Increased CD56+ natural killer cells and related cytokines in major depression. Clin Immunol Immunopathol 1996; 78(1): 83-85.

28. Sluzewska A, Rybakowski J, Bosmans E, Sobieska M, Berghmans R, Maes M, et al. Indicators of immune activation in major depression. Psychiatry Res 1996; 64(3): 161-167.
Address for correspondence:

Masateru Matsushita

Psychiatry, Department of Integrated Medicine Division of Internal Medicine

Osaka University Graduate School of Medicine 2-2-D3 Yamadaoka, Suita

Japan 565-0871

Tel.: +81-6-6879-3055

Fax: +81-6-6879-3059

E-mail: matsushit@psy.med.osaka-u.ac.jp 